\title{
Expression for the Number of Spanning Trees of Line Graphs of Arbitrary Connected Graphs*
}

\author{
Fengming Dong ${ }^{\dagger}$ \\ Mathematics and Mathematics Education \\ National Institute of Education \\ Nanyang Technological University, Singapore 637616 \\ Weigen Yan \\ School of Sciences, Jimei University, Xiamen 361021, China
}

\begin{abstract}
For any graph $G$, let $t(G)$ be the number of spanning trees of $G, L(G)$ be the line graph of $G$ and for any non-negative integer $r, S_{r}(G)$ be the graph obtained from $G$ by replacing each edge $e$ by a path of length $r+1$ connecting the two ends of $e$. In this paper we obtain an expression for $t\left(L\left(S_{r}(G)\right)\right)$ in terms of spanning trees of $G$ by a combinatorial approach. This result generalizes some known results on the relation between $t\left(L\left(S_{r}(G)\right)\right)$ and $t(G)$ and gives an explicit expression $t\left(L\left(S_{r}(G)\right)\right)=k^{m+s-n-1}(r k+2)^{m-n+1} t(G)$ if $G$ is of order $n+s$ and size $m+s$ in which $s$ vertices are of degree 1 and the others are of degree $k$. Thus we prove a conjecture on $t\left(L\left(S_{1}(G)\right)\right)$ for such a graph $G$.
\end{abstract}

Keywords: Graph; Spanning tree; Line graph; Cayley's Foumula; Subdivision.

\section{Introduction}

The graphs considered in this article have no loops but may have parallel edges. For any graph $G$, let $V(G)$ and $E(G)$ be the vertex set and edge set of $G$ respectively, let $S(G)$ be the graph obtained from $G$ by inserting a new vertex to each edge in $G, L(G)$ be the line graph of $G, \mathcal{T}(G)$ be the set of spanning trees of $G$ and $t(G)=|\mathcal{T}(G)|$. Note that for any parallel edges $e$ and $e^{\prime}$ in $G, e$ and $e^{\prime}$ are two vertices in $L(G)$ joined by two parallel edges. For any disjoint subsets $V_{1}, V_{2}$ of $V(G)$, let $E_{G}\left(V_{1}, V_{2}\right)$ (or simply $E\left(V_{1}, V_{2}\right)$ ) denote the set of those edges in $E(G)$ which have ends in $V_{1}$ and $V_{2}$ respectively, and let $E_{G}\left(V_{1}, V(G)-V_{1}\right)$ be simply denoted by $E_{G}\left(V_{1}\right)$. For any $u \in V(G)$, let $E_{G}(u)$ (or simply $E(u)$ ) denote the set $E_{G}(\{u\})$. So the degree of $u$ in $G$, denoted by $d_{G}(u)$ (or simply $d(u)$ ), is equal to $|E(u)|$. For any subset $U$ of $V(G)$, let $G[U]$ denote the subgraph of $G$ induced by $U$ and let $G-U$ denote

\footnotetext{
${ }^{*}$ This paper was partially supported by NSFC (No. 11271307, 11171134 and 11571139) and NIE AcRf (RI 2/12 DFM) of Singapore.

${ }^{\dagger}$ Corresponding author. Email: fengming.dong@nie.edu.sg
} 
the subgraph of $G$ induced by $V(G)-U$. For any $E^{\prime} \subseteq E(G)$, let $G\left[E^{\prime}\right]$ be the spanning subgraph of $G$ with edge set $E^{\prime}, G-E^{\prime}$ be the graph $G\left[E(G)-E^{\prime}\right]$ and $G / E^{\prime}$ be the graph obtained from $G$ by contracting all edges of $E^{\prime}$.

Our paper concerns the relation between $t(G)$ and $t(L(G))$ or $t(L(S(G)))$. Such a relation was first found by Vahovskii [19], then by Kelmans [8] and was rediscovered by Cvetković, Doob and Sachs [7] for regular graphs. They showed that if $G$ is a $k$-regular graph of order $n$ and size $m$, then

$$
t(L(G))=k^{m-n-1} 2^{m-n+1} t(G) .
$$

The first result on the relation between $t(G)$ and $t(L(S(G)))$ was found by Zhang, Chen and Chen [21]. They proved that if $G$ is $k$-regular, then

$$
t(L(S(G)))=k^{m-n-1}(k+2)^{m-n+1} t(G) .
$$

Yan [20] recently generalized the result of (1.1). He proved that if $G$ is a graph of order $n+s$ and size $m+s$ in which $s$ vertices are of degree 1 and all others are of degree $k$, where $k \geq 2$, then

$$
t(L(G))=k^{m+s-n-1} 2^{m-n+1} t(G) .
$$

Yan [20] also proposed a conjecture to generalize the result of (1.2).

Conjecture 1.1 ([20]) Let $G$ be a connected graph of order $n+s$ and size $m+s$ in which $s$ vertices are of degree 1 and all others are of degree $k$. Then

$$
t(L(S(G)))=k^{m+s-n-1}(k+2)^{m-n+1} t(G) .
$$

If $G$ is a digraph, the relation between $t(G)$ and $t(L(G))$ was first obtained by Knuth [9] by an application of the Matrix-Tree Theorem and a bijective proof of the result was found by Bidkhori and Kishore [3]. Note that expressions (1.1), (1.2) and (1.3) were also obtained by the respective authors mentioned above by an application of the Matrix-Tree Theorem. To our knowledge, these results still do not have any combinatorial proofs. Some related results can be seen in [2, 6, 10, 16, 22].

For an arbitrary connected graph $G$ and any non-negative integer $r$, let $S_{r}(G)$ denote the graph obtained from $G$ by replacing each edge $e$ of $G$ by a path of length $r+1$ connecting the two ends of $e$. Thus $S_{0}(G)$ is $G$ itself and $S_{1}(G)$ is the graph $S(G)$. Our main purpose in this paper is to use a combinatorial method to find an expression for $t\left(L\left(S_{r}(G)\right)\right)$ given in Theorem 1.1 .

Theorem 1.1 For any connected graph $G$ and any integer $r \geq 0$,

$$
t\left(L\left(S_{r}(G)\right)\right)=\prod_{v \in V(G)} d(v)^{d(v)-2} \sum_{E^{\prime} \subseteq E(G)} t\left(G\left[E^{\prime}\right]\right) r^{\left|E^{\prime}\right|-|V(G)|+1} \prod_{e \in E(G)-E^{\prime}}\left(d\left(u_{e}\right)^{-1}+d\left(v_{e}\right)^{-1}\right),
$$


where $d(v)=d_{G}(v)$ and $u_{e}$ and $v_{e}$ are the two ends of $e$.

As $S_{0}(G)$ is $G$ itself, the following expression for $t(L(G))$ is a special case of Theorem 1.1 .

$$
t(L(G))=\prod_{v \in V(G)} d(v)^{d(v)-2} \sum_{T \subseteq \mathcal{T}(G)} \prod_{e \in E(G)-E(T)}\left(d\left(u_{e}\right)^{-1}+d\left(v_{e}\right)^{-1}\right) .
$$

The proof of Theorem 1.1 will be completed in Sections 3 and 4. In Section 3, we will show that the case $r=0$ of Theorem 1.1 (i.e., the result (1.5)) is a special case of another result (i.e., Theorem 3.1), and in Section 4, we will prove the case $r \geq 1$ of Theorem 1.1 by applying this theorem for the case $r=0$ (i.e., (1.5)). To establish Theorem 3.1, we need to apply a result in Section 2 (i.e., Proposition 2.3), which determines the number of spanning trees in a graph $G$ with a clique $V_{0}$ such that $F=G-E\left(G\left[V_{0}\right)\right)$ is a forest and every vertex in $V_{0}$ is incident with at most one edge in F. Finally, in Section 5, we will apply Theorem 1.1 to show that for any graph $G$ mentioned in Conjecture 1.1 and any integer $r \geq 0$, we have

$$
t\left(L\left(S_{r}(G)\right)\right)=k^{m+s-n-1}(r k+2)^{m-n+1} t(G) .
$$

Thus (1.3) follows and Conjecture 1.1 is proved.

Note that in the proof of Theorem 1.1, we will express $t\left(L\left(S_{r}(G)\right)\right)$ in another form (i.e., (1.8)), which is actually equivalent to (1.4).

For any graph $G$ and any $E^{\prime} \subseteq E(G)$, let $\Gamma\left(E^{\prime}\right)$ be the set of those mappings $g: E^{\prime} \rightarrow V(G)$ such that for each $e \in E^{\prime}, g(e) \in\left\{u_{e}, v_{e}\right\}$, where $u_{e}$ and $v_{e}$ are the two ends of $e$. Observe that

$$
\sum_{g \in \Gamma\left(E^{\prime}\right)} \prod_{v \in V(G)} d(v)^{-\left|g^{-1}(v)\right|}=\prod_{e \in E^{\prime}}\left(d\left(u_{e}\right)^{-1}+d\left(v_{e}\right)^{-1}\right)
$$

Thus (1.4) and (1.5) can be replaced by the following expressions:

$$
t\left(L\left(S_{r}(G)\right)\right)=\sum_{E^{\prime} \subseteq E(G)} t\left(G\left[E^{\prime}\right]\right) r^{\left|E^{\prime}\right|-|V(G)|+1} \sum_{g \in \Gamma\left(E(G)-E^{\prime}\right)} \prod_{v \in V(G)} d(v)^{d(v)-2-\left|g^{-1}(v)\right|}
$$

and

$$
t(L(G))=\sum_{T \in \mathcal{T}(G)} \sum_{g \in \Gamma(E(G)-E(T))} \prod_{v \in V(G)} d(v)^{d(v)-2-\left|g^{-1}(v)\right|} .
$$

\section{Preliminary Results}

In this section, we shall establish some results which will be used in the next section to prove Theorem 1.1 for the case $r=0$.

For any connected graph $H$ and any forest $F$ of $H$, let $\mathcal{S} \mathcal{T}_{H}(F)$ be the set of those spanning trees of $H$ containing all edges of $F$, and $\mathcal{S F}_{H}(F)$ be the set of those spanning forests of $H$ containing all edges of $F$. 
In this section, we always assume that $G$ is a connected graph with a clique $V_{0}$ such that $F=G-E\left(G\left[V_{0}\right]\right)$ is a forest and every vertex of $V_{0}$ is incident with at most one edge of $F$, as shown in Figure 1

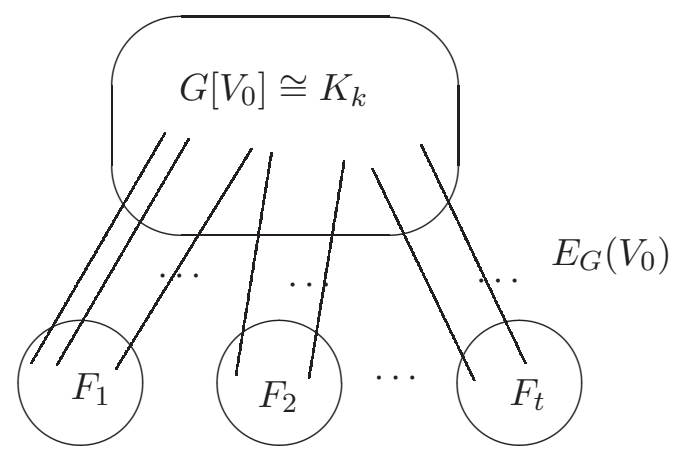

Figure 1: $\quad V_{0}$ is a clique of $G$ such that and $G-E\left(G\left[V_{0}\right]\right)$ is a forest

Let $k=\left|V_{0}\right|, d=\left|E_{G}\left(V_{0}\right)\right|, t=c\left(G-V_{0}\right)$ and $F_{1}, F_{2}, \cdots, F_{t}$ be components of $G-V_{0}$. Observe that $k \geq d \geq t$, as $\left|E_{G}\left(v, V-V_{0}\right)\right| \leq 1$ holds for each $v \in V_{0}$ and $\left|E_{G}\left(V_{0}, V\left(F_{i}\right)\right)\right| \geq 1$ holds for each $F_{i}$.

The main purpose in this section is to show that if $k>d$, then the set $\mathcal{S} \mathcal{T}_{G}(F)$ can be equally partitioned into $\prod_{1 \leq j \leq t}\left|E_{G}\left(V_{0}, V\left(F_{j}\right)\right)\right|$ subsets, each of which has its size $k^{k-2+t-d}$.

In the following, we divide this section into two parts.

\subsection{A preliminary result on trees}

In this subsection, we shall establish some results on trees which are needed for the next subsection and following sections.

Let $T$ be any tree and $V_{0}$ be any proper subset of $V(T)$. Observe that identifying all vertices in $V_{0}$ changes $T$ to a connected graph which is a tree if and only if $\left|E_{T}\left(V_{0}\right)\right|=c\left(T-V_{0}\right)$. So the following observation is obvious.

Lemma 2.1 Let $t=c\left(t-V_{0}\right)$ and $S$ be any proper subset of $E_{T}\left(V_{0}\right)$. Then the two statements below are equivalent:

(i) $\left|S \cap E_{T}\left(V_{0}, V\left(F_{i}\right)\right)\right|=1$ holds for all components $F_{1}, F_{2}, \cdots, F_{t}$ of $T-V_{0}$;

(ii) the graph obtained from $T$ by removing all edges in the set $E_{T}\left(V_{0}\right)-S$ and identifying all vertices of $V_{0}$ is a tree.

With $T, V_{0}$ given above together with a special vertex $v \in V_{0}$ such that $N(v) \subseteq V_{0}$, a subset $S$ of $E_{T}\left(V_{0}\right)$ with the properties in Lemma 2.1 will be determined by a procedure below (i.e., 
Algorithm A). As $S$ is uniquely determined by $T, V_{0}$ and $v$, we can denote it by $\Phi\left(T, V_{0}, v\right)$. Thus $\left|\Phi\left(T, V_{0}, v\right)\right|=t=c\left(T-V_{0}\right)$.

Roughly, if $t=1$, the only edge of $\Phi\left(T, V_{0}, v\right)$ will be selected from $E_{T}\left(V_{0}\right)$ according to the condition that it has one end in the same component of $T\left[V_{0}\right]$ as $v$; if $t \geq 2$, the $t$ edges of $\Phi\left(T, V_{0}, v\right)$ will be determined by the $t-1$ paths $P_{2}, P_{3}, \cdots, P_{t}$ in $T$, where $P_{j}$ is the shortest path connecting vertices of $F_{1}$ and vertices of $F_{j}$ for $j=2,3, \cdots, t$ and $F_{1}, F_{2}, \cdots, F_{t}$ are the components of $T-V_{0}$.

Assume that in Algorithm A, $E(T)=\left\{e_{i}: i \in I\right\}$ for some finite $I$ of positive integers.

Algorithm A with input $\left(T, V_{0}, v\right)$ :

Step A1. Let $t=c\left(T-V_{0}\right)$.

Step A2. If $t=1$, let $\Phi=\left\{e_{j}\right\}$, where $e_{j}$ is the unique edge in the set $E_{T}\left(V_{0}\right)$ which has one end in the component of $T\left[V_{0}\right]$ containing $v$. Go to Step A5.

Step A3. (Now we have $t \geq 2$.)

A3-1. The components of $T-V_{0}$ are labeled as $F_{1}, F_{2}, \cdots, F_{t}$ such that

$$
\min \left\{s: e_{s} \in E_{T}\left(V_{0}, F_{i}\right)\right\}<\min \left\{s^{\prime}: e_{s^{\prime}} \in E_{T}\left(V_{0}, F_{i+1}\right)\right\}
$$

for all $i=1,2, \cdots, t-1$. (In other words, these components are sorted by the minimum edge labels. For example, for the tree $T$ in Figure2(a), the four components $F_{1}, F_{2}, F_{3}, F_{4}$ of $T-V_{0}$ are labeled according to this rule. )

A3-2. For $j=2,3, \cdots, t$, determine the unique path $P_{j}$ in $T$ which is the shortest one among all those paths in $T$ connecting vertices of $F_{1}$ to vertices of $F_{j}$.

Step A4. Let $\Phi=\left(E\left(P_{2}\right) \cap E_{T}\left(V_{0}, V\left(F_{1}\right)\right)\right) \cup \bigcup_{j=2}^{t}\left(E\left(P_{j}\right) \cap E_{T}\left(V_{0}, V\left(F_{j}\right)\right)\right)$.

Step A5. Output $\Phi$.

\section{Remarks:}

(i) Vertex $v$ is needed only for the case that $t=1$;

(ii) If $t=1$, the only edge of $\Phi$ is uniquely determined as $T$ is a tree and $T-V_{0}$ is connected;

(iii) As $T$ is a tree and $F_{1}$ and $F_{j}$ are connected, $P_{j}$ is actually the only path of $T$ with its ends in $F_{1}$ and $F_{j}$ respectively and every internal vertex of $P_{j}$ does not belong to $V\left(F_{1}\right) \cup V\left(F_{j}\right)$. Thus for $P_{j}$ chosen in Step A3,

$$
\left|E\left(P_{j}\right) \cap E_{G}\left(V_{0}, V\left(F_{1}\right)\right)\right|=\left|E\left(P_{j}\right) \cap E_{G}\left(V_{0}, V\left(F_{j}\right)\right)\right|=1,
$$

implying that by Step A4, $\left|\Phi \cap E_{G}\left(V_{0}, V\left(F_{j}\right)\right)\right|=1$ for all $j=1,2, \cdots, t$. 


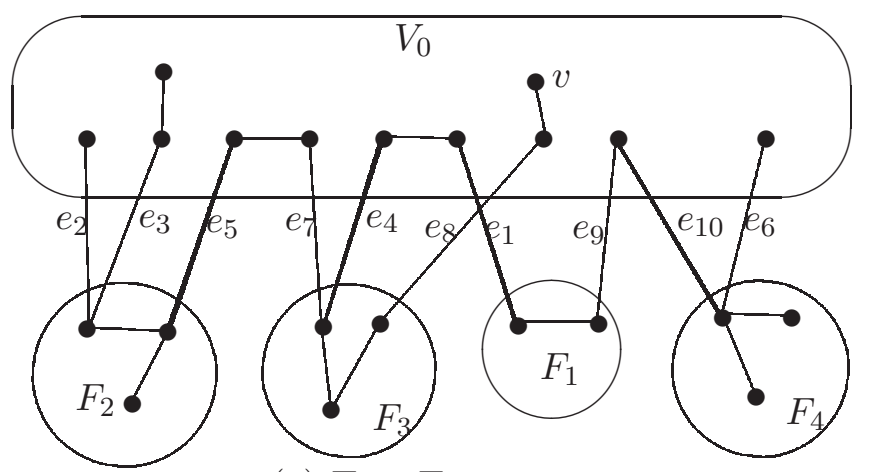

(a) Tree $T$

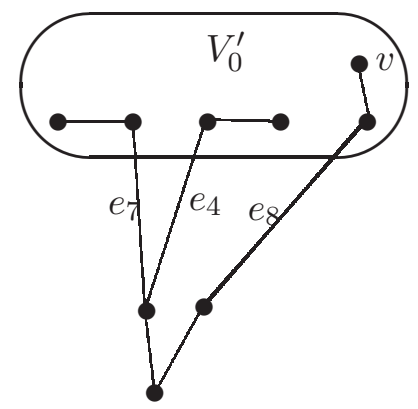

(b) Tree $T^{\prime}$

Figure 2: $\Phi\left(T, V_{0}, v\right)=\left\{e_{1}, e_{4}, e_{5}, e_{10}\right\}$ and $\Phi\left(T^{\prime}, V_{0}^{\prime}, v\right)=\left\{e_{8}\right\}$

For example, for the tree $T$ with $V_{0}$ and $v$ shown in Figure 2 (a), $T-V_{0}$ has four components $F_{1}, F_{2}, F_{3}, F_{4}$ labeled according to the minimum edge labels, and running Algorithm A with input $\left(T, V_{0}, v\right)$ gives $\Phi\left(T, V_{0}, v\right)=\left\{e_{1}, e_{4}, e_{5}, e_{10}\right\}$, as the three paths $P_{2}, P_{3}$ and $P_{4}$ obtained by the algorithm have properties that $\left\{e_{1}, e_{5}\right\} \subseteq E\left(P_{2}\right),\left\{e_{1}, e_{4}\right\} \subseteq E\left(P_{3}\right)$ and $\left\{e_{9}, e_{10}\right\} \subseteq$ $E\left(P_{4}\right)$. For the tree $T^{\prime}$ in Figure 2(b), $T^{\prime}-V_{0}^{\prime}$ has one component only and $\Phi\left(T^{\prime}, V_{0}^{\prime}, v\right)=\left\{e_{8}\right\}$. Note that vertex $v$ is used for finding $\Phi\left(T^{\prime}, V_{0}^{\prime}, v\right)$ but not for finding $\Phi\left(T, V_{0}, v\right)$.

Our second purpose in this subsection is to show that if $\left|E_{T}\left(V_{0}, V\left(F_{j}\right)\right)\right|>1$ for some component $F_{j}$ of $T-V_{0}$, we can find another tree $T^{\prime}$ with $V\left(T^{\prime}\right)=V(T)$ and $T^{\prime}-E\left(T^{\prime}\left[V_{0}\right]\right)=$ $T-E\left(T\left[V_{0}\right]\right)$ such that $\Phi\left(T^{\prime}, V_{0}, v\right)$ and $\Phi\left(T, V_{0}, v\right)$ are different only at choosing the edge joining a vertex of $V_{0}$ to a vertex in $F_{j}$.

For two distinct edges $e, e^{\prime}$ of $E_{T}\left(V_{0}\right)$ incident with $u$ and $u^{\prime}$ respectively, where $u, u^{\prime} \in V_{0}$, let $T\left(e \leftrightarrow e^{\prime}\right)$ be the graph, as shown in Figure 3, obtained from $T$ by changing every edge $(u, w)$ of $T\left[V_{0}\right]$, where $w \neq u^{\prime}$, to $\left(u^{\prime}, w\right)$ and every edge $\left(u^{\prime}, w^{\prime}\right)$ of $T\left[V_{0}\right]$, where $w^{\prime} \neq u$, to $\left(u, w^{\prime}\right)$.

Roughly, $T\left(e \leftrightarrow e^{\prime}\right)$ is actually obtained from $T$ by exchanging $\left(N_{T}(u) \cap V_{0}\right)-\left\{u^{\prime}\right\}$ with $\left(N_{T}\left(u^{\prime}\right) \cap V_{0}\right)-\{u\}$. Note that $u$ and $u^{\prime}$ are adjacent in $T$ if and only if they are adjacent in $T\left(e \leftrightarrow e^{\prime}\right)$.

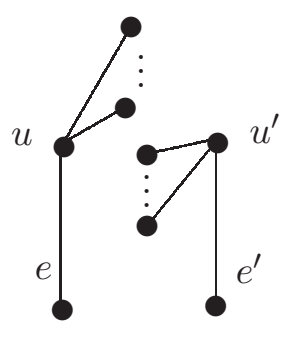

Part of $T$

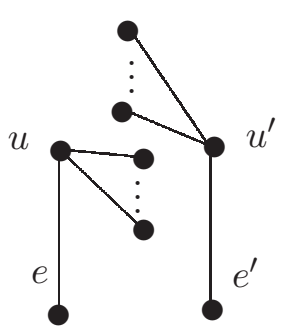

Part of $T\left(e \leftrightarrow e^{\prime}\right)$

Figure 3: $T$ and $T\left(e \leftrightarrow e^{\prime}\right)$

Let $T^{\prime}$ denote $T\left(e \leftrightarrow e^{\prime}\right)$ in the remainder of this subsection. There is a bijection $\tau: E(T) \rightarrow$ 
$E\left(T^{\prime}\right)$ defined below: $\tau(e)=e^{\prime}, \tau\left(e^{\prime}\right)=e, \tau((u, w))=\left(u^{\prime}, w\right)$ whenever $(u, w) \in E(T)$ for $w \in V_{0}-\left\{u^{\prime}\right\}, \tau\left(\left(u^{\prime}, w^{\prime}\right)\right)=\left(u, w^{\prime}\right)$ whenever $\left(u^{\prime}, w^{\prime}\right) \in E(T)$ for $w^{\prime} \in V_{0}-\{u\}$, and $\tau\left(e^{\prime \prime}\right)=e^{\prime \prime}$ for all other edges $e^{\prime \prime}$ in $T$.

Note that $T^{\prime}$ may be not a tree, although $T^{\prime}-V_{0}$ and $T-V_{0}$ are the same graph and $F_{1}, F_{2}, \cdots, F_{t}$ are also the components of $T^{\prime}-V_{0}$. But $T^{\prime}$ is indeed a tree if both $e$ and $e^{\prime}$ have ends in the same component of $T-V_{0}$.

Lemma 2.2 Let $e, e^{\prime}$ be distinct edges of $E_{T}\left(V_{0}, V\left(F_{i}\right)\right)$ for some $i$ with $1 \leq i \leq t$.

(i) Then $T^{\prime}$ is a tree;

(ii) If $e \in \Phi\left(T, V_{0}, v\right)$ and either $t \geq 2$ or $N_{T}(v) \subseteq V_{0}$, then $\Phi\left(T^{\prime}, V_{0}, v\right)=\left(\Phi\left(T, V_{0}, v\right)-\right.$ $\{e\}) \cup\left\{e^{\prime}\right\}$.

Proof. Note that for any edge $e^{\prime \prime} \in E\left(T-V_{0}\right), T / e^{\prime \prime}$ is also a tree, $T^{\prime}$ is a tree if and $T^{\prime} / e^{\prime \prime}$ is a tree, and more importantly, $\Phi\left(T, V_{0}, v\right)=\Phi\left(T / e^{\prime \prime}, V_{0}, v\right)$. Thus it suffices to prove this lemma only for the case that $\left|V\left(F_{i}\right)\right|=1$ for all $i=1,2, \cdots, t$.

(i) It can be proved easily by induction on the number of edges in $T$.

(ii) Assume that $t=1$. Then $N_{T}(v) \subseteq V_{0}$ and so $v$ is not any end of $e$. As $e \in \Phi\left(T, V_{0}, v\right)$, $\Phi\left(T, V_{0}, v\right)=\{e\}$. By Algorithm A, $e$ has one end (i.e., $u$ ) in the component of $T\left[V_{0}\right]$ containing $v$ (i.e., the subgraph $T\left[V_{0}\right]$ has a path $P$ connecting $v$ to $u$ ). By the definition of $T^{\prime}$ (i.e., $\left.T\left(e \leftrightarrow e^{\prime}\right)\right), P$ is now changed to a path $P^{\prime}$ in $T^{\prime}\left[V_{0}\right]$ by the mapping $\tau$ connecting $v$ to one end of $e^{\prime}$ (i.e., $\left.u^{\prime}\right)$. Thus $\Phi\left(T^{\prime}, V_{0}, v\right)=\left\{e^{\prime}\right\}$ by Algorithm A. The result holds for this case.

Now assume that $t \geq 2$. For $j=2,3, \cdots, t$, let $P_{j}$ be the only path in $T$ with its two ends in $F_{1}$ and $F_{j}$ respectively and every interval vertex of $P_{j}$ does not below to $V\left(F_{1}\right) \cup V\left(F_{j}\right)$.

With the bijection $\tau: E(T) \rightarrow E\left(T^{\prime}\right)$ defined above, for $j=2,3, \cdots, t, \tau\left(E\left(P_{j}\right)\right)$ is a subset of $E\left(T^{\prime}\right)$ and forms a path in $T^{\prime}$, denoted by $P_{j}^{\prime}$. Note that the two ends of $P_{j}^{\prime}$ are in $F_{1}$ and $F_{j}$ respectively and every interval vertex of $P_{j}^{\prime}$ does not below to $V\left(F_{1}\right) \cup V\left(F_{j}\right)$. Also observe that for $j=2,3, \cdots, t$, if $i \in\{1, j\}$, then

$$
E\left(P_{j}^{\prime}\right) \cap E_{T^{\prime}}\left(V_{0}, V\left(F_{i}\right)\right)=\left\{e^{\prime}\right\}
$$

and if $s \in\{1, j\}-\{i\}$, then

$$
E\left(P_{j}^{\prime}\right) \cap E_{T^{\prime}}\left(V_{0}, V\left(F_{s}\right)\right)=E\left(P_{j}\right) \cap E_{T}\left(V_{0}, V\left(F_{s}\right)\right) .
$$

Hence (ii) holds. 


\section{$2.2 \quad$ Partitions of $\mathcal{S} \mathcal{T}_{G}(F)$}

Recall that $G$ is a connected graph with a clique $V_{0}$ of order $k$ such that $F=G-E\left(G\left[V_{0}\right]\right)$ is a forest and every vertex of $V_{0}$ is incident with at most one edge of $F$ (i.e., $d_{F}(v) \leq 1$ for each $v \in V_{0}$ ), as shown in Figure 1. In this subsection, our main purpose is to partition $\mathcal{S} \mathcal{T}_{G}(F)$ equally into $\prod_{j=1}^{t}\left|E_{G}\left(V_{0}, V\left(F_{j}\right)\right)\right|$ subsets, where $F_{1}, F_{2}, \cdots, F_{t}$ are the components of $G-V_{0}$.

We start with the following beautiful formula for the number of spanning trees of a complete graph $K_{k}$ of order $k$ containing a given spanning forest. This result was originally due to Lovász (Problem 4 in page 29 of [11]).

Theorem 2.1 ([11]) For any spanning forest $F$ of $K_{k}$, if $c$ is the number of components of $F$ and $k_{1}, k_{2}, \cdots, k_{c}$ are the orders of its components, then

$$
\left|\mathcal{S} \mathcal{T}_{K_{k}}(F)\right|=k^{c-2} \prod_{i=1}^{c} k_{i} .
$$

This result naturally generalizes the well-known formula that $t\left(K_{k}\right)=k^{k-2}$ for any $k \geq 1$, which was first obtained by Cayley [1]. Now we apply this result to establish some results on the set $\mathcal{S T}_{G}(F)$ and finally partition $\mathcal{S T}_{G}(F)$ equally into $\prod_{j=1}^{t}\left|E_{G}\left(V_{0}, V\left(F_{j}\right)\right)\right|$ subsets.

Recall that $d=\left|E_{G}\left(V_{0}\right)\right|$ and $k \geq d \geq t$.

Proposition 2.1 With $G, F$ and $V_{0}$ defined above, we have

$$
\left|\mathcal{S} \mathcal{T}_{G}(F)\right|=k^{k-2+t-d} \prod_{j=1}^{t}\left|E_{G}\left(V_{0}, V\left(F_{j}\right)\right)\right| .
$$

Proof. We only need to consider the case that $E_{G}\left(V_{0}, V\left(F_{j}\right)\right) \neq \emptyset$ for every component $F_{j}$ of $G-V_{0}$; otherwise, the result is trivial as $\left|\mathcal{S} \mathcal{T}_{G}(F)\right|=0$ when $G$ is disconnected.

Observe that for any edge $e$ of $E\left(G-V_{0}\right)$, we have $\left|\mathcal{S} \mathcal{T}_{G / e}(F / e)\right|=\left|\mathcal{S} \mathcal{T}_{G}(F)\right|$. Thus we may assume that every component of $G-V_{0}$ is a single vertex, implying that $G-V_{0}$ is the empty graph of $t$ vertices, namely $x_{1}, x_{2}, \cdots, x_{t}$. So $E(F)=E_{G}\left(V_{0}\right)$.

For each $j=1,2, \cdots, t$, let $V_{j}=\left\{x \in V_{0}: x\right.$ is incident with $\left.x_{j}\right\}$ and $e_{j}$ be any edge joining $x_{j}$ to some vertex in $V_{j}$. Let $G_{0}=G\left[V_{0}\right]$. Note that $F /\left\{e_{1}, e_{2}, \cdots, e_{t}\right\}$ can be considered as a spanning forest of $G_{0}$ and

$$
\mathcal{S T}_{G}(F)=\mathcal{S T}_{G_{0}}\left(F /\left\{e_{1}, e_{2}, \cdots, e_{t}\right\}\right) .
$$

As $G_{0}$ is a complete graph of order $k$, by Theorem 2.1 ,

$$
\left|\mathcal{S} \mathcal{T}_{G_{0}}\left(F /\left\{e_{1}, e_{2}, \cdots, e_{t}\right\}\right)\right|=k^{c-2} \prod_{j=1}^{c}\left|V_{j}^{\prime}\right|,
$$


where $c$ is the number of components of $F /\left\{e_{1}, e_{2}, \cdots, e_{t}\right\}$ and $V_{1}^{\prime}, V_{2}^{\prime}, \cdots, V_{c}^{\prime}$ are vertex sets of components of $F /\left\{e_{1}, e_{2}, \cdots, e_{t}\right\}$. Note that

$$
\left|V_{0}-\bigcup_{j=1}^{t} V_{j}\right|=\left|V_{0}\right|-\sum_{k=1}^{t}\left|V_{j}\right|=k-\left|E_{G}\left(V_{0}\right)\right|=k-d,
$$

implying that $c=k-d+t$ and the sizes of $V_{1}^{\prime}, V_{2}^{\prime}, \cdots, V_{c}^{\prime}$ are equal to

$$
\left|V_{1}\right|, \cdots,\left|V_{t}\right|, \underbrace{1, \cdots, 1}_{k-d} .
$$

As $\left|V_{j}\right|=\left|E_{G}\left(V_{0},\left\{x_{j}\right\}\right)\right|=\left|E_{G}\left(V_{0}, V\left(F_{j}\right)\right)\right|$, the result follows from Theorem 2.1.

Now assume that $v$ is a vertex of $V_{0}$ with $N_{G}(v) \subseteq V_{0}$, i.e., $d_{F}(v)=0$. Note that this condition is only needed for the case that $G-V_{0}$ is connected. Under this condition, it is obvious that $k>d$.

Recall that for any tree $T$ of $\mathcal{S T}_{G}(F), \Phi\left(T, V_{0}, v\right)$ is a subset of $E_{G}\left(V_{0}\right)$ with the property that $\left|\Phi\left(T, V_{0}, v\right) \cap E_{G}\left(V_{0}, V\left(F_{j}\right)\right)\right|=1$ for each $j=1,2, \cdots, t$. For each subset $S$ of $E_{G}\left(V_{0}\right)$ with the property that $\left|S \cap E_{G}\left(V_{0}, V\left(F_{j}\right)\right)\right|=1$ for each $j=1,2, \cdots, t$, let $\mathcal{S T}_{G}(F, S, v)$ denote the set of those spanning trees $T \in \mathcal{S} \mathcal{T}_{G}(F)$ with $\Phi\left(T, V_{0}, v\right)=S$. Thus $\mathcal{S} \mathcal{T}_{G}(F)$ is partitioned into $\prod_{j=1}^{t}\left|E_{G}\left(V_{0}, V\left(F_{j}\right)\right)\right|$ subsets $\mathcal{S} \mathcal{T}_{G}(F, S, v)$ 's. The following result shows that all these sets $\mathcal{S T}_{G}(F, S, v)$ 's have the same size.

The following result shows that $\left|\mathcal{S} \mathcal{T}_{G}(F, S, v)\right|$ is independent of $S$.

Proposition 2.2 Assume that $k>d$ and $N(v) \subseteq V_{0}$. For any subset $S$ of $E_{G}\left(V_{0}\right)$ with $\left|S \cap E_{G}\left(V_{0}, V\left(F_{j}\right)\right)\right|=1$ for each component $F_{j}$ of $G-V_{0}$, we have

$$
\left|\mathcal{S} \mathcal{T}_{G}(F, S, v)\right|=k^{k-2+t-d} \text {. }
$$

Proof. $\quad$ There are exactly $\prod_{j=1}^{t}\left|E_{G}\left(V_{0}, V\left(F_{j}\right)\right)\right|$ subsets $S$ of $E_{G}\left(V_{0}\right)$ with the property that $\left|S \cap E_{G}\left(V_{0}, V\left(F_{j}\right)\right)\right|=1$ for each component $F_{j}$ of $G-V_{0}$. By Proposition 2.1, we only need to show that $\left|\mathcal{S} \mathcal{T}_{G}(F, S, v)\right|=\left|\mathcal{S} \mathcal{T}_{G}\left(F, S^{\prime}, v\right)\right|$ holds for any two such sets $S$ and $S^{\prime}$. Thus it suffices to show that $\left|\mathcal{S} \mathcal{T}_{G}(F, S, v)\right|=\left|\mathcal{S} \mathcal{T}_{G}\left(F, S^{\prime}, v\right)\right|$ holds for any two such sets $S$ and $S^{\prime}$ with $\left|S-S^{\prime}\right|=1$, i.e., $S$ and $S^{\prime}$ have exactly $t-1$ same edges.

Let $S$ be such a subset of $E_{G}\left(V_{0}\right)$ mentioned above. Assume that $e, e^{\prime}$ are distinct edges in $E_{G}\left(V_{0}, V\left(F_{j}\right)\right)$ for some $j$ with $e \in S$ and $e^{\prime} \notin S$. Let $S^{\prime}=(S-\{e\}) \cup\left\{e^{\prime}\right\}$. It remains to show that $\left|\mathcal{S} \mathcal{T}_{G}(F, S, v)\right|=\left|\mathcal{S} \mathcal{T}_{G}\left(F, S^{\prime}, v\right)\right|$.

For any $T \in \mathcal{S} \mathcal{T}_{G}(F, S, v)$, let $T^{\prime}$ be the tree $T\left(e \leftrightarrow e^{\prime}\right)$. By Lemma 2.2, we have $\Phi\left(T^{\prime}, V_{0}, v\right)=$ $\left(\Phi\left(T, V_{0}, v\right)-\{e\}\right) \cup\left\{e^{\prime}\right\}$, implying that $T^{\prime} \in \mathcal{S} \mathcal{T}_{G}\left(F, S^{\prime}, v\right)$.

Let $\phi$ be the mapping from $\mathcal{S T}_{G}(F, S, v)$ to $\mathcal{S} \mathcal{T}_{G}\left(F, S^{\prime}, v\right)$ defined by $\phi(T)=T\left(e \leftrightarrow e^{\prime}\right)$. It is obvious that $\phi$ is an onto mapping, and $\phi^{\prime}: T^{\prime} \rightarrow T^{\prime}\left(e^{\prime} \leftrightarrow e\right)$ is also an onto mapping from $\mathcal{S} \mathcal{T}_{G}\left(F, S^{\prime}, v\right)$ to $\mathcal{S T}_{G}(F, S, v)$. 
Thus $\left|\mathcal{S} \mathcal{T}_{G}(F, S, v)\right|=\left|\mathcal{S} \mathcal{T}_{G}\left(F, S^{\prime}, v\right)\right|$ and the result follows.

We end this section with an application of Proposition 2.2 to deduce another result.

Let $G^{\prime}$ be the graph obtained from $G$ by contracting all edges in $G\left[V_{0}\right]$. Then $V_{0}$ becomes a vertex in $G^{\prime}$, denoted by $v_{0}$. Thus $V\left(G^{\prime}\right)=\left(V(G)-V_{0}\right) \cup\left\{v_{0}\right\}$, and $E\left(G^{\prime}\right)$ and $E(G)-E\left(G\left[V_{0}\right]\right)$ are the same although for each edge $e \in E_{G}\left(V_{0}\right)$, its end in $V_{0}$ is changed to $v_{0}$ when $e$ becomes an edge in $G^{\prime}$. An example for $G$ and $G^{\prime}$ is shown in Figure 4 (a) and (b).

Let $T^{\prime}$ be any spanning tree of $G^{\prime}$ with $E\left(G^{\prime}-v_{0}\right) \subseteq E\left(T^{\prime}\right)$, i.e., $T^{\prime} \in \mathcal{S} \mathcal{T}_{G^{\prime}}\left(F^{\prime}\right)$ for $F^{\prime}=$ $G^{\prime}-v_{0}$. Thus $\left|E_{T^{\prime}}\left(v_{0}\right)\right|=t$, i.e., $E_{T^{\prime}}\left(v_{0}\right)$ has exactly $t$ edges, corresponding to $t$ edges in $G$, one from $E_{G}\left(V_{0}, V\left(F_{j}\right)\right)$ for each component $F_{j}$ of $G-V_{0}$. An example for $T^{\prime}$ is shown in Figure $4(\mathrm{~d})$.

Let $D$ be any subset of $E_{G}\left(V_{0}\right)-E_{T^{\prime}}\left(v_{0}\right)$ and let $\mathcal{S} \mathcal{T}_{G}\left(V_{0}, T^{\prime}, D, v\right)$ be the set of those spanning trees $T$ of $G$ such that (i) $T-V_{0}$ and $T^{\prime}-v_{0}$ are the same graph; (ii) $E_{T}\left(V_{0}\right)$ is the disjoint union of $D$ and $E_{T^{\prime}}\left(v_{0}\right)$ and (iii) $\Phi\left(T, V_{0}, v\right)=E_{T^{\prime}}\left(v_{0}\right)$. Thus $\mathcal{S} \mathcal{T}_{G}\left(V_{0}, T^{\prime}, D, v\right) \subseteq \mathcal{S T}_{G}(F)$ if and only if $D=E_{G}\left(V_{0}\right)-E_{T^{\prime}}\left(v_{0}\right)$. For example, the tree $T$ in Figure 4 (c) belongs to $\mathcal{S} \mathcal{T}_{G}\left(V_{0}, T^{\prime}, D, v\right)$ with $D=\left\{e_{1}, e_{5}\right\}$, but $T \notin \mathcal{S} \mathcal{T}_{G}(F)$, as $E(F) \nsubseteq E(T)$.

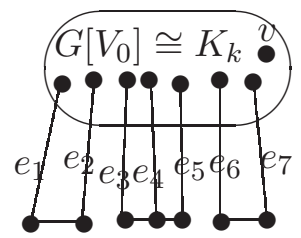

(a) $G$ with $k \geq 8$

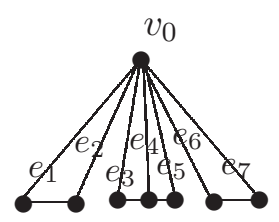

(b) $G^{\prime}$

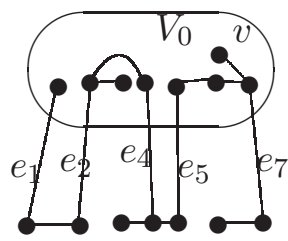

(c) $T$

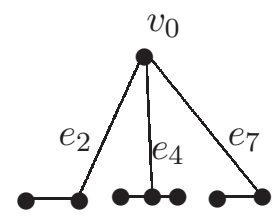

(d) $T^{\prime}$

Figure 4: A tree $T$ in $\mathcal{S T}_{G}\left(V_{0}, T^{\prime}, D, v\right)$ with $D=\left\{e_{1}, e_{5}\right\}$

Proposition 2.3 With $T^{\prime}$ and $D$ given above, we have

$$
\left|\mathcal{S} \mathcal{T}_{G}\left(V_{0}, T^{\prime}, D, v\right)\right|=k^{k-2-|D|} .
$$

Proof. Let $G^{*}$ denote the graph $G-D^{\prime}$, where $D^{\prime}=E_{G}\left(V_{0}\right)-\left(D \cup E_{T^{\prime}}\left(v_{0}\right)\right)$. Observe that

$$
\mathcal{S} \mathcal{T}_{G}\left(V_{0}, T^{\prime}, D, v\right)=\mathcal{S} \mathcal{T}_{G^{*}}\left(F^{*}, E_{T^{\prime}}\left(v_{0}\right), v\right),
$$

where $F^{*}=G^{*}-E\left(G^{*}\left[V_{0}\right]\right)$, i.e., $F^{*}=F-D^{\prime}$. Also note that $c\left(G^{*}-V_{0}\right)=c\left(G-V_{0}\right)=t$ and

$$
\left|E_{G^{*}}\left(V_{0}\right)\right|=\left|E_{T^{\prime}}\left(v_{0}\right) \cup D\right|=t+|D| .
$$

By Proposition 2.2, we have

$$
\left|\mathcal{S} \mathcal{T}_{G^{*}}\left(F^{*}, E_{T^{\prime}}\left(v_{0}\right), v\right)\right|=k^{k-2+t-(t+|D|)}=k^{k-2-|D|} .
$$

Thus the result holds. 


\section{Proving Theorem 1.1 for $r=0$}

In this section, we shall prove Theorem 1.1 for the case $r=0$ (i.e., the result of (1.9) or equivalently (1.5)) is a special case of another result (i.e., Theorem 3.1).

Let $u$ be any vertex in a simple graph $G$. Assume that $E_{G}(u)=\left\{\left(u, u_{i}\right): 1 \leq i \leq s\right\}$, where $s=d_{G}(u)$. If $G^{\prime}$ is the graph obtained from $G-u$ by adding a complete graph $K_{s}$ with vertices $w_{1}, w_{2}, \cdots, w_{s}$ and adding $s$ new edges $\left(w_{i}, u_{i}\right)$ for $i=1,2, \cdots, s$, then $G^{\prime}$ is said to be obtained from $G$ by a clique-insertion at $u$. The clique-insertion is a graph operation playing an important role in the study of vertex-transitive graphs (see [12, 14]). The clique-inserted graph of $G$, denoted by $C(G)$, is obtained from $G$ by operating clique-insertion at every vertex of $G$. Note that the clique-inserted graph of $G$ is also called the para-line graph of $G$ (see [18]). An example for $C(G)$ is shown in Figure 5 .

Let $M$ be the set of those edges in $E(C(G))$ which are not in the inserted cliques. So $M$ consists of all edges in $E(G)$ and thus can be considered as the same as $E(G)$. Observe that $C(G)$ has the following properties:

(i) $M$ is a matching of $C(G)$;

(ii) $L(G)$ is the graph $C(G) / M$ and thus $t(L(G))=\left|\mathcal{S} \mathcal{T}_{C(G)}(M)\right|$;

(iii) each component of $C(G)-M$ is a complete graph.

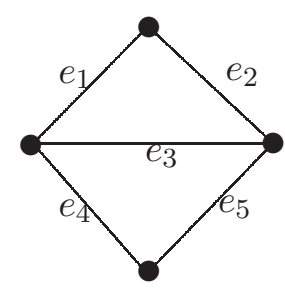

$G$

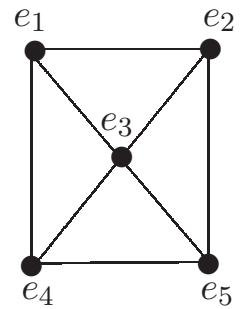

$L(G)$

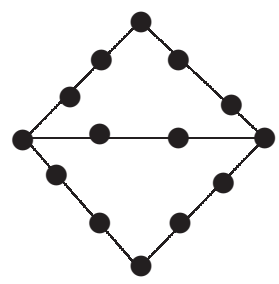

$S_{2}(G)$

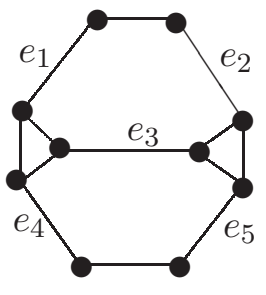

$C(G)$

Figure 5: Line graph $L(G)$ and clique-inserted graph $C(G)$

From observation (iii) above, $C(G)$ is in a type of connected graphs with a matching whose removal yields components which are all complete graphs. As $t(L(G))=\left|\mathcal{S} \mathcal{T}_{C(G)}(M)\right|$ holds for any connected graph $G$ with $M$ defined above, we now extend our problem to finding an expression for $\left|\mathcal{S} \mathcal{T}_{Q}(M)\right|$, where $Q$ is an arbitrary connected graph and $M$ is any matching of $Q$ such that all components of $Q-M$ are complete graphs.

Throughout this section, we assume

(i) $Q$ is a simple and connected graph with a matching $M$ such that all components $Q_{1}, Q_{2}, \cdots, Q_{n}$ of $Q-M$ are complete graphs; 
(ii) for $i=1,2, \cdots, n, V_{i}=V\left(Q_{i}\right)=\left\{v_{i, j}: j=1,2, \cdots, k_{i}\right\}$, where $k_{i}=\left|V_{i}\right|$;

(iii) $M=\left\{e_{1}, e_{2}, \cdots, e_{m}\right\}$ and $M_{i}$ is the set of those edges of $M$ which have one end in $V_{i}$ and $m_{i}=\left|M_{i}\right|$ for $i=1,2, \cdots, n$;

(iv) $v_{i, j}$ is incident with an edge of $M_{i}$ if and only if $1 \leq j \leq m_{i}$;

(v) $Q^{*}$ is the graph obtained from $Q$ by contracting all edges of $Q_{i}$ for all $i=1,2, \cdots, n$. Thus each $Q_{i}$ is converted to a vertex in $Q^{*}$ denoted by $v_{i}$.

With the above assumptions, we observe that $V\left(Q^{*}\right)=\left\{v_{1}, v_{2}, \cdots, v_{n}\right\}$ and $E\left(Q^{*}\right)=M$. As $M$ is a matching of $Q$ and $Q$ is connected, we have $1 \leq m_{i} \leq k_{i}$. If $k_{i}>m_{i}$, then vertex $v_{i, j}$ is not incident with any edge of $M$ for all $j: m_{i}<j \leq k_{i}$. If $k_{i}=m_{i}$ for all $i=1,2, \cdots, n$, then $\left|\mathcal{S} \mathcal{T}_{Q}(M)\right|=t\left(L\left(Q^{*}\right)\right)$. Thus result (1.9) is a special case of Theorem 3.1 which is the main result to be established in this section.

Theorem 3.1 For $Q, Q^{*}$ and $M$ defined above, we have

$$
\left|\mathcal{S T}_{Q}(M)\right|=\sum_{T \in \mathcal{T}\left(Q^{*}\right)} \sum_{f \in \Gamma\left(E\left(Q^{*}\right)-E(T)\right)} \prod_{i=1}^{n} k_{i}^{k_{i}-2-\left|f^{-1}\left(v_{i}\right)\right|} .
$$

To prove Theorem 3.1, by the following result, we only need to consider the case that $k_{i}>m_{i}$ for all $i=1,2, \cdots, n$.

Proposition 3.1 Theorem 3.1 holds if it holds whenever $k_{i}>m_{i}$ for all $i=1,2, \cdots, n$.

Proof. Assume that $M$ is fixed and so all $m_{i}$ 's are fixed. Without loss of generality, we only need to show that with $k_{i}$, where $k_{i} \geq m_{i}$, to be fixed for all $i=2, \cdots, n$, if (3.1) holds for every integer $k_{1}$ with $k_{1} \geq m_{1}+1$, then it also holds for the case $k_{1}=m_{1}$.

For any integer $k_{1} \geq m_{1}$, let

$$
\gamma\left(k_{1}\right)=\left|\mathcal{S} \mathcal{T}_{Q}(M)\right|
$$

By the assumption, for any $k_{1} \geq m_{1}+1$, 3.1) holds and thus

$$
\gamma\left(k_{1}\right)=\sum_{T \in \mathcal{T}\left(Q^{*}\right)} \sum_{f \in \Gamma\left(E\left(Q^{*}\right)-E(T)\right)} k_{1}^{k_{1}-2-\left|f^{-1}\left(v_{1}\right)\right|} \prod_{i=2}^{n} k_{i}^{k_{i}-2-\left|f^{-1}\left(v_{i}\right)\right|}=\sum_{s=0}^{m_{1}-1} a_{s} k_{1}^{k_{1}-2-s},
$$

where

$$
a_{s}=\sum_{T \in \mathcal{T}\left(Q^{*}\right)} \sum_{\substack{f \in \Gamma\left(E\left(Q^{*}\right)-E(T)\right) \\\left|f^{-1}\left(v_{1}\right)\right|=s}} \prod_{i=2}^{n} k_{i}^{k_{i}-2-\left|f^{-1}\left(v_{i}\right)\right|}
$$

It is clear that $a_{s}$ is independent of the value of $k_{1}$. 
Now let $Q^{\prime}$ be the graph $Q-E\left(Q_{1}\right)-\left\{v_{1, j}: m_{1}<j \leq k_{1}\right\}$. So $Q^{\prime}$ is independent of $k_{1}$. Note that for every $T \in \mathcal{S T}_{Q}(M), F=T-E\left(T\left[V_{1}\right]\right)-\left\{v_{1, j}: m_{1}<j \leq k_{1}\right\}$ is a member of $\mathcal{S} \mathcal{F}_{Q^{\prime}}(M)$, i.e., a spanning forest of $Q^{\prime}$ containing all edges of $M$, since $v_{1, j}$ is not incident with any edge of $M$ for all $j: m_{1}<j \leq k_{1}$. Thus $\mathcal{S} \mathcal{T}_{Q}(M)$ can be partitioned into

$$
\mathcal{S} \mathcal{T}_{Q}(M)=\bigcup_{F \in \mathcal{S F}_{Q^{\prime}}(M)} \mathcal{S} \mathcal{T}_{Q^{\prime \prime}}(F),
$$

where $Q^{\prime \prime}=Q\left[E(F) \cup E\left(Q_{1}\right)\right]$. It is possible that $\mathcal{S T}_{Q^{\prime \prime}}(F)=\emptyset$ for some $F \in \mathcal{S} \mathcal{F}_{Q^{\prime}}(M)$. But $\mathcal{S} \mathcal{T}_{Q^{\prime \prime}}\left(F^{\prime}\right) \cap \mathcal{S} \mathcal{T}_{Q^{\prime \prime}}\left(F^{\prime \prime}\right)=\emptyset$ for distinct $F^{\prime}, F^{\prime \prime} \in \mathcal{S F}_{Q^{\prime}}(M)$, implying that for any $k_{1}=\left|V_{1}\right| \geq m_{1}$,

$$
\gamma\left(k_{1}\right)=\sum_{F \in \mathcal{S F}_{Q^{\prime}}(M)}\left|\mathcal{S} \mathcal{T}_{Q^{\prime \prime}}(F)\right| .
$$

By Proposition 2.1, for any $F \in \mathcal{S F}_{Q^{\prime}}(M)$, if $F /\left\{v_{1, j}: 1 \leq j \leq m_{1}\right\}$ is connected, then

$$
\left|\mathcal{S} \mathcal{T}_{Q^{\prime \prime}}(F)\right|=k_{1}^{k_{1}-2+c\left(F-V_{1}\right)-m_{1}} \prod_{j=1}^{c\left(F-V_{1}\right)}\left|E_{F}\left(V_{1}, V\left(F_{j}\right)\right)\right|,
$$

where $F_{1}, F_{2}, \cdots, F_{c\left(F-V_{1}\right)}$ are the components of $F-V_{1}$. Let $\mathcal{S F}_{Q^{\prime}}^{c}(M)$ denote the set of those $F \in \mathcal{S F}_{Q^{\prime}}(M)$ such that $F /\left\{v_{1, j}: 1 \leq j \leq m_{1}\right\}$ is connected. Thus, for any $k_{1} \geq m_{1}$, we have

$$
\begin{aligned}
\gamma\left(k_{1}\right) & =\sum_{F \in \mathcal{S} \mathcal{F}_{Q^{\prime}}^{c}(M)} k_{1}^{k_{1}-2+c\left(F-V_{1}\right)-m_{1}} \prod_{j=1}^{c\left(F-V_{1}\right)}\left|E_{F}\left(V_{1}, V\left(F_{j}\right)\right)\right| \\
& =\sum_{s=0}^{m_{1}-1} b_{s} k_{1}^{k_{1}-2-s},
\end{aligned}
$$

where

$$
b_{s}=\sum_{\substack{F \in \mathcal{S F}^{c} Q^{\prime}(M) \\ c\left(F-V_{1}\right)=m_{1}-s}} \prod_{j=1}^{c\left(F-V_{1}\right)}\left|E_{F}\left(V_{1}, V\left(F_{j}\right)\right)\right| .
$$

As $Q^{\prime}$ is independent of $k_{1}$, for any $F \in \mathcal{S F}_{Q^{\prime}}^{c}(M)$, the expression $\prod_{j=1}^{c\left(F-V_{1}\right)}\left|E_{F}\left(V_{1}, V\left(F_{j}\right)\right)\right|$ is independent of $k_{1}=\left|V_{1}\right|$ and hence $b_{s}$ is independent of $k_{1}$.

By (3.2) and (3.4), for every integer $k_{1}$ with $k_{1} \geq m_{1}+1$, we have

$$
\sum_{s=0}^{m_{1}-1} a_{s} k_{1}^{k_{1}-2-s}=\sum_{s=0}^{m_{1}-1} b_{s} k_{1}^{k_{1}-2-s}
$$

where $a_{s}$ and $b_{s}$ are independent of $k_{1}$ for all $s=0,1,2, \cdots, m_{1}-1$. Considering sufficiently large values of $k_{1}$ in (3.6), we come to the conclusion that $a_{s}=b_{s}$ for all $s=0,1, \cdots, m_{1}$, implying that

$$
\begin{aligned}
\gamma\left(m_{1}\right) & =\sum_{s=0}^{m_{1}-1} b_{s} m_{1}^{m_{1}-2-s}=\sum_{s=0}^{m_{1}-1} a_{s} m_{1}^{m_{1}-2-s} \\
& =\sum_{T \in \mathcal{T}\left(Q^{*}\right)} \sum_{f \in \Gamma\left(E\left(Q^{*}\right)-E(T)\right)} m_{1}^{m_{1}-2-\left|f^{-1}\left(v_{1}\right)\right|} \prod_{i=2}^{n} k_{i}^{k_{i}-2-\left|f^{-1}\left(v_{i}\right)\right|}
\end{aligned}
$$


implying that (3.1) holds for $k_{1}=m_{1}$. Hence the result holds.

In the remainder of this section, we assume that $k_{i} \geq m_{i}+1$ for all $i$ with $1 \leq i \leq n$. Thus vertex $v_{i, k_{i}}$ is not incident with any edge of $M$ for each $i$. We will complete the proof of Theorem 3.1 by the approach explained in the two steps below:

(a) $\mathcal{S} \mathcal{T}_{Q}(M)$ will be partitioned into $t\left(Q^{*}\right) 2^{m-n+1}$ subsets denoted by $\Delta\left(T_{0}, f\right)$ 's, corresponding to $t\left(Q^{*}\right) 2^{m-n+1}$ ordered pairs $\left(T_{0}, f\right)$, where $T_{0} \in \mathcal{T}\left(Q^{*}\right)$ and $f \in \Gamma\left(E\left(Q^{*}\right)-E\left(T_{0}\right)\right)$;

(b) then we show that for any given $T_{0} \in \mathcal{T}\left(Q^{*}\right)$ and $f \in \Gamma\left(E\left(Q^{*}\right)-E\left(T_{0}\right)\right)$,

$$
\left|\Delta\left(T_{0}, f\right)\right|=\prod_{i=1}^{n} k_{i}^{k_{i}-2-\left|f^{-1}\left(v_{i}\right)\right|} .
$$

Step (a) above will be done by Algorithm B below which determines a spanning tree $T_{0}$ of $Q^{*}$ and a mapping $f \in \Gamma\left(E\left(Q^{*}\right)-E\left(T_{0}\right)\right)$ for any given $T \in \mathcal{S} \mathcal{T}_{Q}(M)$.

Algorithm B $\left(T \in \mathcal{S} \mathcal{T}_{Q}(M)\right)$ :

Step B1. Let $T_{n}$ be $T$;

Step B2. for $i=n, n-1, \cdots, 1$, let $D_{i}=E_{T_{i}}\left(V_{i}\right)-\Phi\left(T_{i}, V_{i}, v_{i, k_{i}}\right)$ and $T_{i-1}$ be the graph obtained from $T_{i}$ by deleting all edges in $D_{i} \cup E\left(T_{i}\left[V_{i}\right]\right)$ and identifying all vertices of $V_{i}$ as one, denoted by $v_{i}$, which is a vertex of $Q^{*}$;

Step B3. output $T_{0}$ and $f$, where $f$ is a mapping from $D_{1} \cup D_{2} \cup \cdots \cup D_{n}$ to $V\left(Q^{*}\right)$ defined by $f(e)=v_{i}$ whenever $e \in D_{i}$.

By Lemma 2.1, each graph $T_{i}$ produced in the process of running Algorithm B is indeed a tree and thus $T_{0}$ is a tree in $\mathcal{T}\left(Q^{*}\right)$. It is also clear that $D_{1} \cup D_{2} \cup \cdots \cup D_{n}=E\left(Q^{*}\right)-E\left(T_{0}\right)$ and so the mapping $f$ output by Algorithm B belongs to $\Gamma\left(E\left(Q^{*}\right)-E\left(T_{0}\right)\right)$.

An example is presented below. Let $T$ be a tree in $\mathcal{S} \mathcal{T}_{Q}(M)$ as shown in Figure 6(a), where $Q$ is a connected graph with a matching $M=\left\{e_{1}, e_{2}, \cdots, e_{8}\right\}$ such that $Q-M$ has four components $Q_{1}, Q_{2}, Q_{3}$ and $Q_{4}$ isomorphic to complete graphs of orders $5,4,6,5$ respectively. If we run Algorithm $\mathrm{B}$ with this tree $T$ as its input, then we have $T_{3}, T_{2}, T_{1}$ and $T_{0}$ as shown in Figure 6 and thus

$$
D_{4}=\left\{e_{4}\right\}, D_{3}=\left\{e_{1}, e_{2}\right\}, D_{2}=\left\{e_{5}, e_{7}\right\}, D_{1}=\emptyset,
$$

implying that the mapping $f \in \Gamma\left(E\left(Q^{*}\right)-E\left(T_{0}\right)\right)$ output by Algorithm B, where $E\left(Q^{*}\right)-$ $E\left(T_{0}\right)=\left\{e_{1}, e_{2}, e_{4}, e_{5}, e_{7}\right\}$, is the one given below:

$$
f\left(e_{1}\right)=f\left(e_{2}\right)=v_{3}, f\left(e_{4}\right)=v_{4}, f\left(e_{5}\right)=f\left(e_{7}\right)=v_{2} .
$$




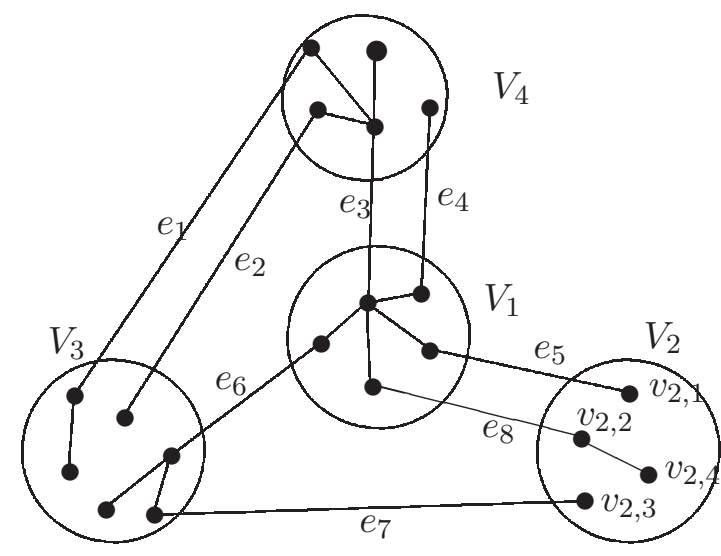

(a) $T \in \mathcal{S T}_{G}(M)$ (i.e., $T_{4}$ )

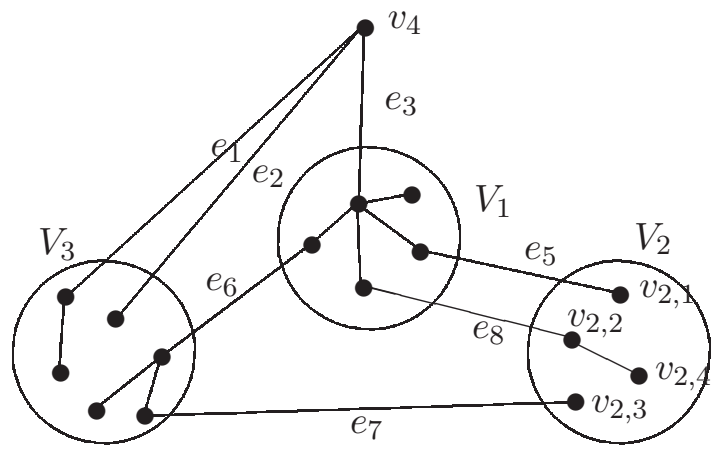

(b) $T_{3}$

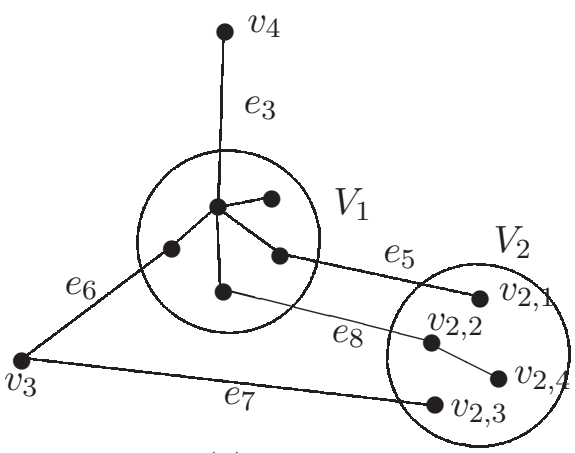

(c) $T_{2}$

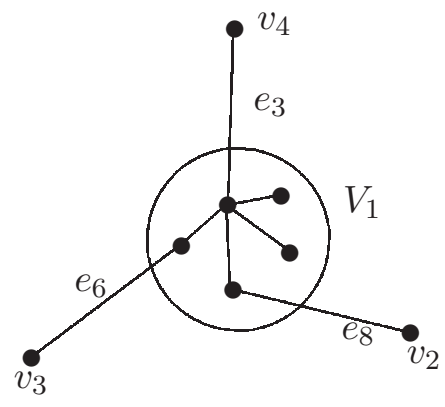

(d) $T_{1}$

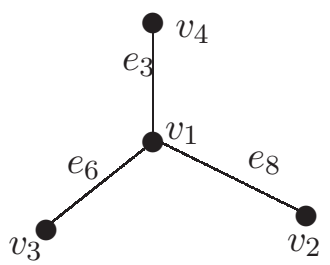

(e) $T_{0}$

Figure 6: $T \in \mathcal{S} \mathcal{T}_{Q}(M)$ (i.e., $\left.T_{4}\right)$ and $T_{3}, T_{2}, T_{1}, T_{0}$

Let $\psi$ be a mapping from $\mathcal{S} \mathcal{T}_{Q}(M)$ to the following set of ordered pair $\left(T_{0}, f\right)$ 's:

$$
\left\{\left(T_{0}, f\right): T_{0} \in \mathcal{T}\left(Q^{*}\right), f \in \Gamma\left(E(Q)-E\left(T_{0}\right)\right)\right\}
$$

defined by $\psi(T)=\left(T_{0}, f\right)$ if $T_{0}$ and $f$ are output by running Algorithm $B$ with input $T$. For any $T_{0} \in \mathcal{T}\left(Q^{*}\right)$ and $f \in \Gamma\left(E(Q)-E\left(T_{0}\right)\right)$, let $\Delta\left(T_{0}, f\right)=\psi^{-1}\left(T_{0}, f\right)$. Thus $\mathcal{S} \mathcal{T}_{Q}(M)$ is partitioned into $t\left(Q^{*}\right) 2^{m-n+1}$ subsets $\Delta\left(T_{0}, f\right)$ 's, where $T_{0} \in \mathcal{T}\left(Q^{*}\right)$ and $f \in \Gamma\left(E(Q)-E\left(T_{0}\right)\right)$.

The proof of Theorem 3.1 now remains to determine the size of $\Delta\left(T_{0}, f\right)$ below.

Proposition 3.2 For any $T_{0} \in \mathcal{T}\left(Q^{*}\right)$ and $f \in \Gamma\left(E\left(Q^{*}\right)-E\left(T_{0}\right)\right)$, we have

$$
\left|\Delta\left(T_{0}, f\right)\right|=\prod_{i=1}^{n} k_{i}^{k_{i}-2-\left|f^{-1}\left(v_{i}\right)\right|} .
$$

Proof. Let $D_{i}=f^{-1}\left(v_{i}\right)=\left\{e \in M-E\left(T_{0}\right): f(e)=v_{i}\right\}$ for $i=1,2, \cdots, n$. So $D_{i} \subseteq M_{i}$. By Algorithm $B, T$ is a member of $\Delta\left(T_{0}, f\right)$ if and only if there exist trees $T_{1}, T_{2}, \cdots, T_{n-1}$ such that for $i=n, n-1, \cdots, 1$, the following properties hold, where $T_{n}$ is the tree $T$ :

(P1) $V\left(T_{i}\right)=\left(V\left(T_{i-1}\right)-\left\{v_{i}\right\}\right) \cup V_{i}$; 
(P2) $T_{i}-V_{i}$ and $T_{i-1}-v_{i}$ are the same graph; and

(P3) $E_{T_{i-1}}\left(v_{i}\right)=\Phi\left(T_{i}, V_{i}, v_{i, k_{i}}\right)=E_{T_{i}}\left(V_{i}, V\left(T_{i}\right)-V_{i}\right)-D_{i}$ and $D_{i} \subseteq E_{T_{i}}\left(V_{i}, V\left(T_{i}\right)-V_{i}\right)$.

Let $U_{i}=\bigcup_{1 \leq j \leq i} V_{j} \cup\left\{v_{i+1}, \cdots, v_{n}\right\}$. Observe that if properties (P1), (P2) and (P3) hold for all $i$ with $1 \leq i \leq n$, then $V\left(T_{i}\right)=U_{i}$ for all $i=0,1, \cdots, n$.

Now let $\Delta_{0}=\left\{T_{0}\right\}$. Define sets $\Delta_{1}, \Delta_{2}, \cdots, \Delta_{n}$ as follows. For $i=1,2, \cdots, n$, let

$$
\Delta_{i}=\bigcup_{T_{i-1} \in \Delta_{i-1}} \Psi\left(T_{i-1}\right)
$$

where $\Psi\left(T_{i-1}\right)$ is the set of all those spanning trees $T_{i}$ of $H_{i}$ such that properties (P1), (P2) and (P3) hold for $T_{i}$ and $T_{i-1}$ and $H_{i}$ is the graph with $V\left(H_{i}\right)=U_{i}$ such that $V_{i}$ is a clique of $H_{i}, H_{i}-V_{i}$ is the same as $T_{i-1}-v_{i}$ and $E_{H_{i}}\left(V_{i}\right)=E_{T_{i-1}}\left(v_{i}\right) \cup D_{i}$. Note that for each edge $e \in E_{H_{i}}\left(V_{i}\right)$, $e$ is actually also an edge in $Q$ and we assume that $e$ joins the same pair of vertices as it does in $Q$ unless $e$ as an edge of $Q$ has one end in some $V_{j}$ with $j>i$, while in this case this end of $e$ in $H_{i}$ is $v_{j}$.

By (P1), (P2) and (P3), $T_{i-1}$ is uniquely determined by any $T_{i} \in \Psi\left(T_{i-1}\right)$. Thus $\Psi\left(T_{i-1}^{\prime}\right) \cap$ $\Psi\left(T_{i-1}^{\prime \prime}\right)=\emptyset$ for any distinct members $T_{i-1}^{\prime}$ and $T_{i-1}^{\prime \prime}$ of $\Delta_{i-1}$. For any $T_{i-1} \in \Delta_{i-1}$, observe that $\Psi\left(T_{i-1}\right)$ is actually the set $\mathcal{S} \mathcal{T}_{H_{i}}\left(V_{i}, T_{i-1}, D_{i}, v_{i, k_{i}}\right)$, and thus by Proposition 2.3 , we have

$$
\left|\Psi\left(T_{i-1}\right)\right|=k_{i}^{k_{i}-2-\left|D_{i}\right|} .
$$

Hence $\left|\Delta_{i}\right|=k_{i}^{k_{i}-2-\left|D_{i}\right|}\left|\Delta_{i-1}\right|$ for all $i=1,2, \cdots, n$. As $\Delta\left(T_{0}, f\right)=\Delta_{n}$, the result holds.

We end this section with a proof of Theorem 3.1 .

Proof of Theorem 3.1. By Proposition 3.1, we may assume that $k_{i}>m_{i}$ for all $i=1,2, \cdots, n$. By the definition of $\psi$ and $\Delta\left(T_{0}, f\right)=\psi^{-1}\left(T_{0}, f\right)$, we have

$$
\mathcal{S} \mathcal{T}_{Q}(M)=\bigcup_{\substack{T_{0} \in \mathcal{T}(H) \\ f \in \Delta\left(E(H)-E\left(T_{0}\right)\right)}} \Delta\left(T_{0}, f\right)
$$

where the union gives a partition of $\mathcal{S} \mathcal{T}_{Q}(M)$. Thus

$$
\left|\mathcal{S} \mathcal{T}_{Q}(M)\right|=\sum_{\substack{T_{0} \in \mathcal{T}(H) \\ f \in \Gamma\left(E(H)-E\left(T_{0}\right)\right)}}\left|\Delta\left(T_{0}, f\right)\right|=\sum_{\substack{T_{0} \in \mathcal{T}(H) \\ f \in \Gamma\left(E(H)-E\left(T_{0}\right)\right)}} \prod_{i=1}^{n} k_{i}^{k_{i}-2-\left|f^{-1}\left(v_{i}\right)\right|}
$$

where the last step follows from Proposition 3.2 . Hence Theorem 3.1 holds.

\section{Proving Theorem 1.1 for $r \geq 1$}

In this section, we shall prove Theorem 1.1 for the case $r \geq 1$. 
For any graph $G$ and edge $e$ in $G$, let $G-e$ and $G / e$ be the graphs obtained from $G$ by deleting $e$ and contracting $e$ respectively. The following result is obvious.

Lemma $4.1([4,5])$ For any graph $G$ and edge e in $G$, we have

$$
t(G)=t(G-e)+t(G / e) .
$$

In particular, if e is a bridge of $G$, then $t(G)=t(G / e)$.

For any edge $e$ in $G$, let $G_{\bullet}$ be the graph obtained from $G$ by inserting a vertex on $e$ and $G_{-e}$ be the graph obtained from $G-e$ by attaching a pendent edge to each end of $e$, as shown in Figure 7. Similarly, for any $E^{\prime} \subseteq E(G)$, let $G_{\bullet E^{\prime}}$ be the graph obtained from $G$ by inserting a vertex on each edge of $E^{\prime}$ and $G_{-E^{\prime}}$ be the graph obtained from $G-E^{\prime}$ by attaching a pendent edge to each end of $e$ for all $e \in E^{\prime}$. Clearly $G_{\bullet} E^{\prime}$ is the graph $S(G)$ when $E^{\prime}=E(G)$.

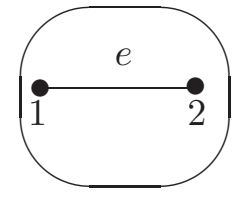

(a)

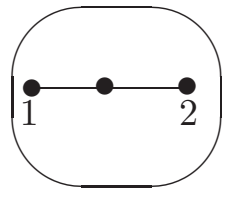

(b)

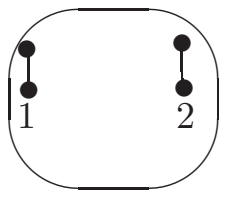

(c)

Figure 7: (a) $G$ with edge $e \quad$ (b) The graph $G_{\bullet} \quad$ (c) The graph $G_{-e}$

By the definition of the line graph, the following lemma follows from Lemma 4.1.

Lemma 4.2 Let $G$ be any graph and e be an edge in $G$. Then

$$
t\left(L\left(G_{\bullet e}\right)\right)=t(L(G))+t\left(L\left(G_{-e}\right)\right) .
$$

In particular, if e is a bridge of $G$, then $t\left(L\left(G_{\bullet}\right)\right)=t(L(G))$.

For any edge $e$ in $G$ and any non-negative integer $r$, let $G_{r \bullet e}$ be the graph obtained from $G$ by inserting $r$ new vertices on $e$, i.e., replacing $e$ by a path of length $r+1$ connecting the two ends of $e$. For any subset $F$ of $E(G)$, let $G_{r \bullet F}$ be the graph obtained from $G$ by replacing each edge $e$ of $F$ by a path of length $r+1$ connecting the two ends of $e$.

Lemma 4.3 Let $G$ be any graph and $F$ be any subset of $E(G)$. Then, for any $r \geq 0$,

$$
t\left(L\left(G_{r \bullet F}\right)\right)=\sum_{E^{\prime} \subseteq F} r^{\mid E^{\prime}} t\left(L\left(G_{-E^{\prime}}\right)\right)
$$


Proof. Note that for any two vertices $u, v$ in a graph $H$, if $N_{H}(u)=\{v\}$ and $d_{H}(v)=2$, then $t(L(H))=t(L(H-u))$. Thus, for any edge $e$ of $G$ and any positive integer $r$, by Lemma 4.2, we have

$$
t\left(L\left(G_{r \bullet e}\right)\right)=t\left(L\left(G_{(r-1) \bullet e}\right)\right)+t\left(L\left(G_{-e}\right)\right),
$$

where $G_{0 \bullet e}$ is $G$. Applying (4.2) repeatedly deduces that

$$
t\left(L\left(G_{r \bullet e}\right)\right)=t(L(G))+r t\left(L\left(G_{-e}\right)\right) .
$$

Note that (4.1) is obvious for $F=\emptyset$ or $r=0$. Now assume that $e \in F$ and $r \geq 1$. By induction, we have

$$
t\left(L\left(G_{r \bullet F-\{e\}}\right)\right)=\sum_{E^{\prime} \subseteq F-\{e\}} r^{\left|E^{\prime}\right|} t\left(L\left(G_{-E^{\prime}}\right)\right)
$$

By (4.3), we have

$$
t\left(L\left(G_{r \bullet F}\right)\right)=t\left(L\left(G_{r \bullet F-\{e\}}\right)\right)+r t\left(L\left(\left(G_{r \bullet F-\{e\}}\right)_{-e}\right)\right) .
$$

Thus (4.1) follows immediately from (4.4).

We are now ready to prove Theorem 1.1 for the case $r \geq 1$.

Proof of Theorem 1.1 for $r \geq 1$ : Assume that $r \geq 1$. By Lemma 4.3, we have

$$
t\left(L\left(S_{r}(G)\right)\right)=\sum_{E^{\prime} \subseteq E(G)} r^{\left|E^{\prime}\right|} t\left(L\left(G_{-E^{\prime}}\right)\right) .
$$

The above summation needs only to take those subsets $E^{\prime}$ of $E(G)$ with $t\left(L\left(G_{-E^{\prime}}\right)\right)>0$ (i.e. $G-E^{\prime}$ is connected). Now let $E^{\prime}$ be any fixed subset of $E(G)$ such that $G-E^{\prime}$ is connected and let $H$ denote $G_{-E^{\prime}}$. By Theorem 1.1 for $r=0$ (i.e., (1.9)),

$$
t\left(L\left(G_{-E^{\prime}}\right)\right)=\sum_{T^{\prime} \in \mathcal{T}(H)} \sum_{g \in \Gamma\left(E(H)-E\left(T^{\prime}\right)\right)} \prod_{v \in V(H)} d_{H}(v)^{d_{H}(v)-2-\left|g^{-1}(v)\right|} .
$$

Observe that $V(G) \subseteq V(H)$. For any $v \in V(H)$, if $v \in V(G)$, then $d_{H}(v)=d_{G}(v)$; otherwise, $d_{H}(v)=1$. Thus

$$
\prod_{v \in V(H)} d_{H}(v)^{d_{H}(v)-2-\left|g^{-1}(v)\right|}=\prod_{v \in V(G)} d_{G}(v)^{d_{G}(v)-2-\left|g^{-1}(v)\right|} .
$$

For each $T^{\prime} \in \mathcal{T}(H), T^{\prime}$ contains all pendent edges in $H$ and so $T^{\prime}$ corresponds to $T$, where $T=T^{\prime}[V(G)]$, which is a spanning tree of $G-E^{\prime}$. Thus $E(H)-E\left(T^{\prime}\right)=E\left(G-E^{\prime}\right)-E(T)$ and

$$
t\left(L\left(G_{-E^{\prime}}\right)\right)=\sum_{T \in \mathcal{T}\left(G-E^{\prime}\right)} \sum_{g \in \Gamma\left(E\left(G-E^{\prime}\right)-E(T)\right)} \prod_{v \in V(G)} d_{G}(v)^{d_{G}(v)-2-\left|g^{-1}(v)\right|} .
$$

By (4.6) and (4.9),

$$
t\left(L\left(S_{r}(G)\right)\right)=\sum_{E^{\prime} \in E(G)} r^{\left|E^{\prime}\right|} \sum_{T \in \mathcal{T}\left(G-E^{\prime}\right)} \sum_{g \in \Gamma\left(E\left(G-E^{\prime}\right)-E(T)\right)} \prod_{v \in V(G)} d_{G}(v)^{d_{G}(v)-2-\left|g^{-1}(v)\right|} .
$$


By replacing $E(G)-E^{\prime}-E(T)$ by $E^{\prime \prime}$, (4.10) implies that

$$
\begin{aligned}
& t\left(L\left(S_{r}(G)\right)\right)=\sum_{E^{\prime \prime} \subseteq E(G)} \sum_{T^{\prime} \in \mathcal{T}\left(G-E^{\prime \prime}\right)} \sum_{g \in \Gamma\left(E^{\prime \prime}\right)} r^{|E(G)|-\left|E^{\prime \prime}\right|-\left|E\left(T^{\prime}\right)\right|} \prod_{v \in V(G)} d_{G}(v)^{d_{G}(v)-2-\left|g^{-1}(v)\right|} \\
& =\sum_{E^{\prime \prime} \subseteq E(G)} r^{|E(G)|-\left|E^{\prime \prime}\right|-|V(G)|+1} t\left(G-E^{\prime \prime}\right) \sum_{g \in \Gamma\left(E^{\prime \prime}\right)} \prod_{v \in V(G)} d_{G}(v)^{d_{G}(v)-2-\left|g^{-1}(v)\right|} \\
& =\sum_{E^{\prime \prime \prime} \subseteq E(G)} r^{\left|E^{\prime \prime \prime}\right|-|V(G)|+1} t\left(G\left[E^{\prime \prime \prime}\right]\right) \sum_{g \in \Gamma\left(E-E^{\prime \prime \prime}\right)} \prod_{v \in V(G)} d_{G}(v)^{d_{G}(v)-2-\left|g^{-1}(v)\right|} .
\end{aligned}
$$

Hence the case $r \geq 1$ of Theorem 1.1 holds.

\section{$5 \quad$ Proof of Conjecture 1.1}

Now we turn back to those connected graphs $G$ mentioned in Conjecture 1.1 and apply the following result and Theorem 1.1 to deduce a relation between $t\left(L\left(S_{r}(G)\right)\right)$ and $t(G)$. The case $r=1$ of this relation is exactly the conclusion of Conjecture 1.1.

Lemma 5.1 Let $H$ be any connected graph of order $n$ and size $m$. For any integer $i$ with $0 \leq i \leq m-n+1$, we have

$$
\left(\begin{array}{c}
m-n+1 \\
i
\end{array}\right) t(H)=\sum_{\substack{\prime \\
E^{\prime} \subseteq E(H) \\
\left|E^{\prime}\right|=i}} t\left(H-E^{\prime}\right) .
$$

Proof. We prove this result by providing two different methods to determining the size of the following set:

$$
\Theta=\left\{\left(T, E^{\prime}\right): T \text { is a spanning tree of } H \text { and } E^{\prime} \subseteq E(H)-E(T) \text { with }\left|E^{\prime}\right|=i\right\} .
$$

Note that for each spanning tree $T$ of $H$, as $|E(H)|=m$ and $|E(T)|=n-1$, the number of subsets $E^{\prime}$ of $E(H)-E(T)$ with $\left|E^{\prime}\right|=i$ is $\left(\begin{array}{c}m-n+1 \\ i\end{array}\right)$. On the other hand, for each $E^{\prime} \subseteq E(H)$ with $\left|E^{\prime}\right|=i$, there are exactly $t\left(H-E^{\prime}\right)$ spanning trees $T$ of $G$ such that $E^{\prime} \subseteq E(H)-E(T)$. Thus the result holds.

We now deduce the following consequence of Theorem 1.1 for those connected graphs $G$ mentioned in Conjecture 1.1 .

Corollary 5.1 Let $G$ be a connected graph of order $n+s$ and size $m+s$ in which $s$ vertices are of degree 1 and all others are of degree $k$, where $k \geq 2$. Then, for any $r \geq 0$,

$$
t\left(L\left(S_{r}(G)\right)\right)=k^{m+s-n-1}(r k+2)^{m-n+1} t(G) .
$$


Proof. For any $E^{\prime} \subseteq E(G)$ with $t\left(G\left[E^{\prime}\right]\right) \neq 0, E^{\prime}$ contains every bridge of $G$, and so $d\left(u_{e}\right)=d\left(v_{e}\right)=k$ for all $e \in E(G)-E^{\prime}$. By Theorem 1.1, we have

$$
\begin{aligned}
t\left(L\left(S_{r}(G)\right)\right) & =\left(k^{k-2}\right)^{n} \sum_{E^{\prime} \subseteq E(G)} t\left(G\left[E^{\prime}\right]\right) r^{\left|E^{\prime}\right|-(n+s)+1}\left(2 k^{-1}\right)^{(m+s)-\left|E^{\prime}\right|} \\
& =\left(k^{k-2}\right)^{n} r^{-(n+s)+1}\left(2 k^{-1}\right)^{(m+s)} \sum_{E^{\prime} \subseteq E(G)} t\left(G\left[E^{\prime}\right]\right) r^{\left|E^{\prime}\right|}\left(2 k^{-1}\right)^{-\left|E^{\prime}\right|} \\
& =\left(k^{k-2}\right)^{n} r^{-(n+s)+1}\left(2 k^{-1}\right)^{(m+s)} \sum_{E^{\prime \prime} \subseteq E(G)} t\left(G-E^{\prime \prime}\right) r^{|E(G)|-\left|E^{\prime \prime}\right|}\left(2 k^{-1}\right)^{\left|E^{\prime \prime}\right|-|E(G)|} \\
& =\left(k^{k-2}\right)^{n} r^{-(n+s)+1}\left(2 k^{-1}\right)^{(m+s)} \sum_{j=0}^{m-n+1} r^{m+s-j}\left(2 k^{-1}\right)^{j-m-s} \sum_{E^{\prime \prime} \subseteq E(G)} t\left(G-E^{\prime \prime}\right) \\
& =\left(k^{\prime \prime \prime} \mid=j\right. \\
& =\left(k^{k-2}\right)^{n} \sum_{j=0}^{m-n+1} r^{m-n+1-j}\left(2 k^{-1}\right)^{j}\left(m-2 k^{-1}\right)^{m-n+1} t(G) \\
& =k^{n(k-2)-(m-n+1)}(k r+2)^{m-n+1} t(G) \\
& =k^{m+s-n-1}(k r+2)^{m-n+1} t(G),
\end{aligned}
$$

where the last expression follows from the equality $2(m+s)=k n+s$ by the given conditions on $G$. Hence the result is obtained.

Notice that (1.3) is the special case of Corollary 5.1 for $r=0$ while the conclusion of Conjecture 1.1 is the special case of Corollary 5.1 for $r=1$.

We end this section with the the following result on some special bipartite graphs, which can be obtained by applying Lemma 5.1 and the case $r=0$ of Theorem 1.1 .

Corollary 5.2 Let $G=(A, B ; E)$ be a connected bipartite graph of order $n$ and size $m$ such that $d(x) \in\{1, a\}$ for all $x \in A$ and $d(y) \in\{1, b\}$ for all $y \in B$, where $a \geq 2$ and $b \geq 2$. Then

$$
t(L(G))=a^{(a-2) n_{1}} b^{(b-2) n_{2}}\left(a^{-1}+b^{-1}\right)^{m-n+1} t(G),
$$

where $n_{1}$ is the number of vertices $x$ in $A$ with $d(x)=a$ and $n_{2}$ is the number of vertices $y$ in $B$ with $d(y)=b$.

The result of Corollary 5.2 in the case that $G$ is an $(a, b)$-semiregular bipartite graph was originally due to Cvetković (see Theorem 3.9 in [13], $§ 5.2$ of [15], or [17]).

Acknowledgement. The authors wish to thank the referees for their very helpful suggestions. 


\section{References}

[1] M. Aigner and G. Ziegler, Proofs from The Book, Fourth edition. Springer-Verlag, Berlin, 2010.

[2] A. Berget, A. Manion, M. Maxwell, A. Potechin, V. Reiner, The critical group of a line graph, Ann. Comb. 16 (2012), 449-488.

[3] H. Bidkhori and S. Kishore, Counting spanning trees of a directed line graph, arXiv: $0910.3442 \mathrm{v} 1$.

[4] N. L. Biggs, Algebraic Graph Theory, 2nd edn, Cambridge, Cambridge University Press, 1993.

[5] J. A. Bondy and U. S. R. Murty, Graph Theory with Applications, American Elsevier, New York, 1976.

[6] H. Y. Chen, F. J. Zhang, The critical group of a clique-inserted graph, Discrete Math. 319 (2014), 24-32.

[7] D.Cvetković, M.Doob, H.Sachs, Spectra of Graphs. Theory and Application, Pure Appl. Math.,vol. 87, Academic Press,Inc. [Harcourt Brace Jovanovich, Publishers], New York, London,1980.

[8] A.K.Kelmans, On properties of the characteristic polynomial of a graph, in:Kibernetiku Na Sluzbu Kom., vol.4, Gosener-goizdat, Moscow, 1967, pp.27-47(in Russian).

[9] D.E. Knuth. Oriented subtrees of an arc digraph, J. of Combin. Theory 3 (1967), 309314.

[10] L. Levine, Sandpile groups and spanning trees of directed line graphs, J. of Combin. Theory Ser. A 118 (2011), 350-364.

[11] L. Lovász, Combinatorial Problems and Exercises, North-Holland, Amsterdam (1979).

[12] L. Lovász, M.D. Plummer, Matching Theory, Ann. Discrete Math. 29, North-Holland, Amsterdam, 1986.

[13] I.G. Macdonald, Symmetric functions and Hall polynomials, 2nd edition. Oxford Mathematical Monographs. Oxford Science Publications. The Clarendon Press, Oxford University Press, New York, 1995.

[14] W. Mader, Minimale $n$-fach kantenzusammenhangende Graphen, Math. Ann. 191 (1971), 21-28. 
[15] B. Mohar, The Laplacian Spectrum of Graphs, Graph Theory, Combinatorics, and Applications 2 Ed. by Y. Alavi, G. Chartrand, O. R. Oellermann, A. J. Schwenk. Wiley, 1991, 871-898.

[16] D. Perkinson, N. Salter, T. Y. Xu, A note on the critical group of a line graph, Electron. J. Combin. 18 (2011), \#P124.

[17] I. Sato, Zeta functions and complexities of a semiregular bipartite graph and its line graph, Discrete Math. 307 (2007), 237-245.

[18] T. Shirai, The spectrum of infinite regular line graphs, Trans. Amer. Math. Soc. 352 (2000), no. 1, 115-132.

[19] E.B.Vahovskii, On the characteristic numbers of incidence matrices for non-singular graphs, Sibirsk. Mat. Zh. 6 (1965), 44-49 (in Russian).

[20] Weigen Yan, On the number of spanning trees of some irregular line graphs, J. Combin. Theory Ser. A 120 (2013), 1642-1648.

[21] F.J.Zhang, Y.-C.Chen, Z.B.Chen, Clique-inserted-graphs and spectral dynamics of clique-inserting, J. Math. Anal. Appl. 349 (2009), 211-225.

[22] Z. H. Zhang, Some physical and chemical indices of clique-inserted lattices, Journal of Statistical Mechanics: Theory and Experiment, doi:10.1088/1742-5468/2013/10/P10004. 